\title{
Complexity of LTL Model-Checking for Safe Object Nets
}

\author{
Michael Köhler-Bußmeier and Frank Heitmann \\ University of Hamburg, Department for Informatics \\ Vogt-Kölln-Straße 30, D-22527 Hamburg \\ koehler, heitmann@informatik.uni-hamburg.de
}

\begin{abstract}
In this paper we present recently obtained results from [14 concerning the complexity of LTL model checking of safe Elementary Object Nets (EOs) in a novel and more algorithmic oriented way.

Object nets are Petri nets which have Petri nets as tokens - an approach known as the nets-within-nets paradigm. Object nets are called elementary if the net system has a two levelled structure. Due to these two modelling levels object nets are very suited to model the mobility of e.g. active objects or agents. The well known $\mathrm{p} / \mathrm{t}$ nets can be viewed as a special case of Eos.

For $\mathrm{p} / \mathrm{t}$ nets the concept of safeness means that there is at most one token on each place. Since object nets have nested markings there are different possibilities to generalise this idea for Eos. In this paper we concentrate on the variant of Eos safeness that guarantees the finiteness of state spaces and show that for safe EOs the LTL model checking problem is PSPACE-complete.
\end{abstract}

\section{Model Checking for Object Nets}

In the following we investigate the analysis of object nets using temporal logics. Object nets are Petri nets which have Petri nets as tokens - an approach which is called the nets-within-nets paradigm, proposed by Valk [22, 24] for a two levelled structure and generalised in [11, 12] for arbitrary nesting structures. ${ }^{1}$ The Petri nets that are used as tokens are called net-tokens. Net-tokens are tokens with internal structure and inner activity. Due to these two modelling levels object nets are very suited to model the mobility of active objects or agents (cf. 9] and [10]).

In [12, 8] we studied decidability properties of unbounded object nets. In this paper we repeat very recent results from [14] concerning bounded object nets and present them in a novel way. In particular we repeat the formalism of (safe) elementary object nets and sharpen the definitions in some points. We then prove that checking if a safe Eos satisfies a property expressed in LTL is PSPACE-complete. This was first proved in [14. We recapitulate the proof here, but take a more algorithmic oriented point of view and thus make the proof more elegant and easier to follow in several places. Throughout this paper we stress the relevance of the presented modelling formalism for the modelling of mobile objects and agents.

The paper has the following structure: In Section 2 elementary object systems (Eos) are defined. In section 3 safe Eos are defined and in section 4 we discuss the complexity of LTL model checking these nets. The paper ends with a conclusion.

In the following we assume basic knowledge of Petri nets, see e.g. [20].

\footnotetext{
${ }^{1}$ Related approaches dealing with nesting and Petri nets are object nets [23], recursive nets [4, nested nets [17, $\mathrm{PN}^{2}$ [5, MOB nets [15, hypernets [1, Mobile Systems [16], AHO systems [6], adaptive workflow nets [18], and Hornets [13.
} 


\section{Elementary Object Systems}

An elementary object system (Eos) is composed of a system net, which is a $\mathrm{p} / \mathrm{t}$ net $\widehat{N}=$ $(\widehat{P}, \widehat{T}$, pre, post $)$ and a set of object nets $\mathcal{N}=\left\{N_{1}, \ldots, N_{n}\right\}$, which are $\mathrm{p} / \mathrm{t}$ nets given as $N=\left(P_{N}, T_{N}\right.$, pre $_{N}$, post $\left._{N}\right)$. We assume that all sets of nodes (places and transitions) are pairwise disjoint. Moreover we have $\widehat{N} \notin \mathcal{N}$ and we assume the existence of the object net - $\in \mathcal{N}$ which has no places and no transitions and is used to model anonymous, so called black tokens.

Figure 1 shows an example, which will be explained in detail in example 2.2 and 2.4 below. One may imagine the object nets (the nets which reside on places of the system net) to model mobile agents with internal structure and activity, while the system net models the environment in which the agents act and interact.

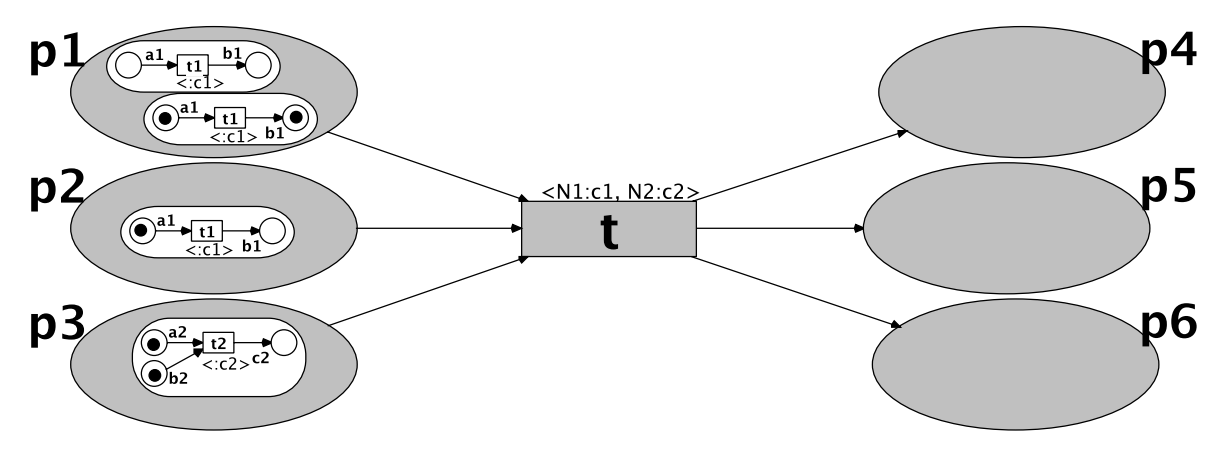

Figure 1: An object net

The system net places are typed by the mapping $d: \widehat{P} \rightarrow \mathcal{N}$ with the meaning, that the place $\widehat{p}$ of the system net contains net-tokens of the object net type $N$ if $d(\widehat{p})=N .^{2}$ No place of the system net is mapped to the system net itself, since $\widehat{N} \notin \mathcal{N}$.

Since the tokens of an Eos are instances of object nets a marking $\mu \in \mathcal{M}$ of an Eos $O S$ is a nested multiset. A marking of an EOS $O S$ is denoted $\mu=\sum_{k=1}^{|\mu|}\left(\widehat{p}_{k}, M_{k}\right)$ where $\widehat{p}_{k}$ is a place in the system net and $M_{k}$ is the marking of the net-token of type $d\left(\widehat{p}_{k}\right)$. To emphasise the nesting, markings are also denoted as $\mu=\sum_{k=1}^{|\mu|} \widehat{p}_{k}\left[M_{k}\right]$. Tokens of the form $\widehat{p}[\mathbf{0}]$ and $d(\widehat{p})=\bullet$ are abbreviated as $\widehat{p}[]$.

The set of all markings which are syntactically consistent with the typing $d$ is denoted $\mathcal{M}$ (Here $d^{-1}(N) \subseteq \widehat{P}$ is the set of system net places of the type $N$ ):

$$
\mathcal{M}:=M S\left(\bigcup_{N \in \mathcal{N}}\left(d^{-1}(N) \times M S\left(P_{N}\right)\right)\right)
$$

The transitions in an Eos are labelled with synchronisation channels. We assume a fixed set of channels $C$, including the channel $\epsilon$ which is used to describe the absence of any "real" channel. Each transition of the system net has one label for each object, defined by the labelling function function $\widehat{l}: \widehat{T} \rightarrow(N \rightarrow C)$. Each transition of an object net $N$ has one single label,

\footnotetext{
${ }^{2}$ In the following the terms (marked) object net and net-token are used almost interchangeable. We use the term net-token whenever we like to emphasise the aspect that the marked object net is a token of the system net.
} 
defined by the labelling function function $l_{N}: T_{N} \rightarrow C .^{3}$

A system event is generated by transitions with matching labels. The labelling allows three cases of events:

1. System-autonomous firing: Whenever $\widehat{l}(\widehat{t})(N)=\epsilon$ holds for all $N \in \mathcal{N}$, the system net transition $\widehat{t}$ fires autonomously.

2. Synchronised firing: There is at least one object net that has to be synchronised, i.e. there is a $N$ such that $\widehat{l}(\widehat{t})(N) \neq \epsilon$.

3. Object-autonomous firing: An object net $N$ 's transition $t$ fires autonomously whenever $l_{N}(t)=\epsilon$.

These three kinds of events can be reduced to the case of a synchronisation where a system net transition has exactly one synchronisation partner in each object net. This normal form is obtained by adding some idle transitions: For each object net $N \in \mathcal{N}$ we add the idle-transitions $\epsilon_{N}$ with pre $_{N}\left(\epsilon_{N}\right)=$ post $_{N}\left(\epsilon_{N}\right)=\mathbf{0}$ to its transition set. For object-autonomous events we also add the set of idle transitions $\epsilon_{\widehat{P}}:=\left\{\epsilon_{\widehat{p}} \mid \widehat{p} \in \widehat{P}\right\}$ with $\operatorname{pre}\left(\epsilon_{\widehat{p}}\right)=\operatorname{post}\left(\epsilon_{\widehat{p}}\right)=\widehat{p}$ to the set of system net transitions. We extend the labelling to idle transitions by $\widehat{l}\left(\epsilon_{\widehat{p}}\right)(N)=l_{N}\left(\epsilon_{N}\right)=\epsilon$ for all $\widehat{p} \in \widehat{P}$ and $N \in \mathcal{N}$.

With these idle transitions the channel $\epsilon$ (which means "no synchronisation") is modelled as a synchronisation with an idle transition that has no effect.

The synchronisation labelling generates the set of system events $\Theta$. An event is a pair denoted $\widehat{\tau}[\theta]$ in the following. Here, $\widehat{\tau}$ is either a real transition $\widehat{t}$ or $\epsilon_{\widehat{p}}$ for some $\widehat{p} ; \theta$ maps each object net to one of its transitions. An event has the meaning that the system net transition $\widehat{\tau}$ fires synchronously with all the object net transitions $\theta(N), N \in \mathcal{N}$.

A special case for the mapping $\theta$ is the idle map $\epsilon_{\mathcal{N}}$ which is defined $\epsilon_{\mathcal{N}}(N)=\epsilon_{N}$ for all $N \in \mathcal{N}$.

All events are generated from the labels: $\widehat{l}(\widehat{\tau})(N)=l_{N}(\theta(N))$ must hold for all $N \in \mathcal{N}$. Whenever $\widehat{\tau}$ is an idle transition $\epsilon_{\widehat{p}} \in \epsilon_{\widehat{P}}$ we also demand that $\theta(N)$ is the idle event $\epsilon_{N}$ except for exactly one object net $N$ (which is the object-autonomous event), i.e. $\mid\{N \in \mathcal{N}: \theta(N) \neq$ $\left.\epsilon_{N}\right\} \mid=1$ holds.

$$
\begin{aligned}
& \Theta_{l}:=\{\widehat{\tau}[\theta] \mid \forall N \in \mathcal{N}: \widehat{l}(\widehat{t})(N)=l_{N}(\theta(N)) \wedge \\
&\left.\widehat{\tau} \in \epsilon_{\widehat{P}} \Longrightarrow\left|\left\{N \in \mathcal{N}: \theta(N) \neq \epsilon_{N}\right\}\right|=1\right\}
\end{aligned}
$$

Definition 2.1 (EOS). An elementary object system (EOS) is a tuple $O S=\left(\widehat{N}, \mathcal{N}, d, \Theta_{l}\right)$ such that:

1. $\widehat{N}$ is a $p / t$ net, called the system net.

2. $\mathcal{N}$ is a finite set of disjoint $p / t$ nets, called object nets.

3. $d: \widehat{P} \rightarrow \mathcal{N}$ is the typing of the system net places.

4. $\Theta_{l}$ is the set of events generated from the labelling $l=\left(\widehat{l},\left(l_{N}\right)_{N \in \mathcal{N}}\right)$.

An Eos with initial marking is a tuple $O S=\left(\widehat{N}, \mathcal{N}, d, \Theta_{l}, \mu_{0}\right)$ where $\mu_{0} \in \mathcal{M}$ is the initial marking.

\footnotetext{
${ }^{3}$ In the graphical representation the synchronisation labelling is defined by transition inscriptions in the form $\left\langle N_{1}: \widehat{l}(\widehat{t})\left(N_{1}\right), \ldots\right\rangle$ in the system net and in the form $\left\langle: l_{N}(t)\right\rangle$ in the object nets $(\epsilon$ is omitted).
} 
Example 2.2. Figure 1 shows an EOS with the system net $\widehat{N}$ and the object nets $\mathcal{N}=\left\{N_{1}, N_{2}\right\}$. The system net is given as $\widehat{N}=(\widehat{P}, \widehat{T}$, pre, post $)$ with $\widehat{P}=\left\{p_{1}, \ldots, p_{6}\right\}$ and $\widehat{T}=\{t\}$. The first object net is $N_{1}=\left(P_{1}, T_{1}\right.$, pre $_{1}$, post pr $\left._{1}\right)$ with $P_{1}=\left\{a_{1}, b_{1}\right\}$ and $T_{1}=\left\{t_{1}\right\}$. The second object net is $N_{2}=\left(P_{2}, T_{2}\right.$, pre $_{2}$, post pos $\left._{2}\right)$ with $P_{2}=\left\{a_{2}, b_{2}, c_{2}\right\}$ and $T_{2}=\left\{t_{2}\right\}$. The typing is $d\left(p_{1}\right)=d\left(p_{2}\right)=d\left(p_{4}\right)=N_{1}$ and $d\left(p_{3}\right)=d\left(p_{5}\right)=d\left(p_{6}\right)=N_{2}$. The labelling function of the system net $\widehat{l}$ is defined by $\widehat{l}(t)\left(N_{1}\right)=c_{1}$ and $\widehat{l}(t)\left(N_{2}\right)=c_{2}$. The labelling $l_{N_{1}}$ of the first object net is defined by setting $l_{N_{1}}\left(t_{1}\right)=c_{1}$. Similarily, $l_{N_{2}}$ is defined by $l_{N_{2}}\left(t_{2}\right)=c_{2}$. There is only one synchronous event: $\Theta_{l}=\left\{t\left[N_{1} \mapsto t_{1}, N_{2} \mapsto t_{2}\right]\right\}$. The initial marking has two net-tokens on $p_{1}$, one on $p_{2}$, and one on $p_{3}$ :

$$
\mu=p_{1}\left[a_{1}+b_{1}\right]+p_{1}[\mathbf{0}]+p_{2}\left[a_{1}\right]+p_{3}\left[a_{2}+b_{2}\right]
$$

Note that in figure 1 the structure of the three net-tokens is the same on $p_{1}$ and $p_{2}$ but the nettokens' markings are different. Note also that the object nets may be viewed as models of mobile agents with internal activity. The system net then models the environment. ${ }^{4}$ In example 2.5 below a mobile agent, which resides in a slightly more sophisticated environment, is presented. $\diamond$

We name special properties of Eos: An Eos is $p / t$-like iff it has only places for black tokens: $d(\widehat{P})=\{\bullet\}$. An Eos is a generalised state machine (GSM) iff for all $\widehat{t}$ there is either exactly one place in the preset and one in the postset typed with the same object net $N$ or there are no such places:

$$
\forall N \in \mathcal{N}: \forall \widehat{t} \in \widehat{T}:|\{\widehat{p} \in \bullet \widehat{t} \mid d(\widehat{p})=N\}|=|\{\widehat{p} \in \widehat{t} \bullet \mid d(\widehat{p})=N\}| \leq 1
$$

and the inital marking has at most one net-token of each type:

$$
\forall N \in \mathcal{N}: \sum_{\widehat{p} \in \widehat{P}, d(\widehat{p})=N} \Pi^{1}\left(\mu_{0}\right)(\widehat{p}) \leq 1
$$

Obviously each $\mathrm{p} / \mathrm{t}$-like EOS is a GSM since $d(\widehat{p})=\bullet$ for all $\widehat{p}$.

\subsection{Projections and Firing Rule}

Firing a system event $\widehat{\tau}[\theta] \in \Theta_{l}$ removes net-tokens together with their individual internal markings. A nested multiset $\lambda \in \mathcal{M}$ that is part of the current marking $\mu$, i.e. $\lambda \leq \mu$, is replaced by a nested multiset $\rho$.

Let $\mu=\sum_{k=1}^{|\mu|}\left(\widehat{p}_{k}, M_{k}\right)$ be a marking of an Eos. To formalize the firing rule we need to introduce projections. The projection $\Pi^{1}$ on the first component abstracts away the substructure of all net-tokens:

$$
\Pi^{1}\left(\sum_{k=1}^{|\mu|} \widehat{p}_{k}\left[M_{k}\right]\right):=\sum_{k=1}^{|\mu|} \widehat{p}_{k}
$$

The projection $\Pi_{N}^{2}$ on the second component is the abstract marking of all net-tokens of the type $N \in \mathcal{N}$ ignoring their local distribution within the system net:

$$
\Pi_{N}^{2}\left(\sum_{k=1}^{|\mu|} \widehat{p}_{k}\left[M_{k}\right]\right):=\sum_{k=1}^{|\mu|} \mathbf{1}_{N}\left(\widehat{p}_{k}\right) \cdot M_{k}
$$

\footnotetext{
${ }^{4}$ Since we only have one system net transition, the system net in figure 1 should be viewed as an excerpt of a larger system net only.
} 
where the indicator function $\mathbf{1}_{N}: \widehat{P} \rightarrow\{0,1\}$ is given by $\mathbf{1}_{N}(\widehat{p})=1$ iff $d(\widehat{p})=N$. Note that $\Pi_{N}^{2}(\mu)$ results in an marking of the object net $N$.

The enabling condition is expressed by the enabling predicate $\phi_{O S}$ (or just $\phi$ whenever $O S$ is clear from the context):

$$
\begin{aligned}
\phi(\widehat{\tau}[\theta], \lambda, \rho) & \Longleftrightarrow \Pi^{1}(\lambda)=\operatorname{pre}(\widehat{\tau}) \wedge \Pi^{1}(\rho)=\operatorname{post}(\widehat{\tau}) \wedge \\
& \forall N \in \mathcal{N}: \Pi_{N}^{2}(\lambda) \geq \operatorname{pre}_{N}(\theta(N)) \wedge \\
& \forall N \in \mathcal{N}: \Pi_{N}^{2}(\rho)=\Pi_{N}^{2}(\lambda)-\operatorname{pre}_{N}(\theta(N))+\operatorname{post}_{N}(\theta(N))
\end{aligned}
$$

With $\widehat{M}=\Pi^{1}(\lambda)$ and $\widehat{M}^{\prime}=\Pi^{1}(\rho)$ as well as $M_{N}=\Pi_{N}^{2}(\lambda)$ and $M_{N}^{\prime}=\Pi_{N}^{2}(\rho)$ for all $N \in \mathcal{N}$ the predicate $\phi$ has the following meaning:

1. The first conjunct expresses that the system net multiset $\widehat{M}$ corresponds to the precondition of the system net transition $\widehat{\tau}$, i.e. $\widehat{M}=\operatorname{pre}(\widehat{\tau})$.

2. In turn, a multiset $\widehat{M}^{\prime}$ is produced, that corresponds to the post-set of $\widehat{\tau}$.

3. An object net transition $t_{N}$ is enabled if the combination $M_{N}$ of the markings of net-tokens of type $N$ enables it, i.e. $M_{N} \geq$ pre $_{N}(\theta(N))$.

4. The firing of $\widehat{\tau}[\theta]$ must also obey the object marking distribution condition

$$
M_{N}^{\prime}=M_{N}-\operatorname{pre}_{N}(\theta(N))+\text { post }_{N}(\theta(N))
$$

where post $_{N}(\theta(N))-$ pre $_{N}(\theta(N))$ is the effect of the object net's transition on the nettokens.

Note that (1) and (2) assures that only net-tokens relevant for the firing are included in $\lambda$ and $\rho$. Conditions (3) and (4) allows for additonal tokens in the net-tokens.

For system-autonomous events $\widehat{\tau}\left[\epsilon_{\mathcal{N}}\right]$ the enabling predicate $\phi$ can be simplified further. We have $\operatorname{pre}_{N}\left(\epsilon_{N}\right)=\operatorname{post}_{N}\left(\epsilon_{N}\right)=\mathbf{0}$. This ensures $\Pi_{N}^{2}(\lambda)=\Pi_{N}^{2}(\rho)$, i.e. the sum of markings in the copies of a net-token is preserved w.r.t. each type $N$. This condition ensures the existence of linear invariance properties (cf. [12, Prop. 7).

Analogously, for an object-autonomous event we have an idle-transition $\widehat{\tau}=\epsilon_{\widehat{p}}$ for the system net and the first and the second conjunct is: $\Pi^{1}(\lambda)=\operatorname{pre}(\widehat{t})=\widehat{p}=\operatorname{post}(\widehat{t})=\Pi^{1}(\rho)$. So, there is an addend $\lambda=\widehat{p}[M]$ in $\mu$ with $d(\widehat{p})=N$ and $M$ enables $t_{N}:=\theta(N)$.

Definition 2.3 (Firing Rule). Let $O S$ be an Eos and $\mu, \mu^{\prime} \in \mathcal{M}$ markings. The event $\widehat{\tau}[\theta]$ is enabled in $\mu$ for the mode $(\lambda, \rho) \in \mathcal{M}^{2}$ iff $\lambda \leq \mu \wedge \phi(\widehat{\tau}[\theta], \lambda, \rho)$ holds.

An event $\widehat{\tau}[\theta]$ that is enabled in $\mu$ for the mode $(\lambda, \rho)$ can fire: $\mu \stackrel{\widehat{\tau}[\theta](\lambda, \rho)}{O S} \mu^{\prime}$. The resulting successor marking is defined as $\mu^{\prime}=\mu-\lambda+\rho$.

We write $\mu \underset{O S}{\stackrel{\widehat{\tau}[\theta]}{\longrightarrow}} \mu^{\prime}$ whenever $\mu \stackrel{\widehat{\tau}[\theta](\lambda, \rho)}{O S} \mu^{\prime}$ for some mode $(\lambda, \rho)$.

Example 2.4. Consider the Eos of figure 1 again. The current marking $\mu$ of the Eos enables $t\left[N_{1} \mapsto t_{1}, N_{2} \mapsto t_{2}\right]$ in the mode $(\lambda, \rho)$ where

$$
\begin{aligned}
\lambda & =p_{1}\left[a_{1}+b_{1}\right]+p_{2}\left[a_{1}\right]+p_{3}\left[a_{2}+b_{2}\right] \\
\rho & =p_{4}\left[a_{1}+b_{1}+b_{1}\right]+p_{5}[\mathbf{0}]+p_{6}\left[c_{2}\right]
\end{aligned}
$$




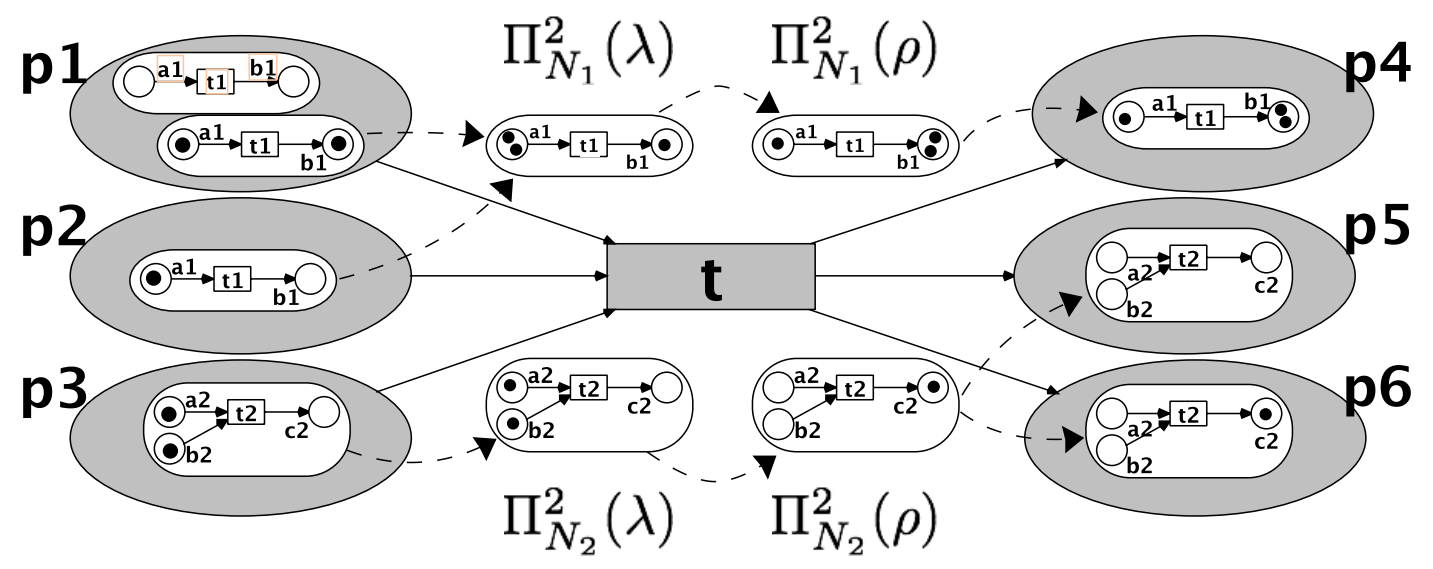

Figure 2: The EOS of figure 1 illustrating the projections $\Pi_{N}^{2}(\lambda)$ and $\Pi_{N}^{2}(\rho)$

We have the current marking:

$$
\mu=p_{1}[\mathbf{0}]+\underbrace{p_{1}\left[a_{1}+b_{1}\right]+p_{2}\left[a_{1}\right]+p_{3}\left[a_{2}+b_{2}\right]}_{\lambda}=p_{1}[\mathbf{0}]+\lambda
$$

The net-tokens' markings are added by the projections $\Pi_{N}^{2}$, resulting in the markings $\Pi_{N}^{2}(\lambda)$. The sub-synchronisation generate $\Pi_{N}^{2}(\rho)$. (The results are shown above and below the transition t.) After the synchronisation we obtain the successor marking on $p_{4}, p_{5}$, and $p_{6}$ as shown in figure 2:

$$
\begin{aligned}
\mu^{\prime} & =(\mu-\lambda)+\rho=p_{1}[\mathbf{0}]+\rho \\
& =p_{1}[\mathbf{0}]+p_{4}\left[a_{1}+b_{1}+b_{1}\right]+p_{5}[\mathbf{0}]+p_{6}\left[c_{2}\right]
\end{aligned}
$$

Note that a different successor marking is possible: $\rho^{\prime}=p_{4}\left[a_{1}+b_{1}+b_{1}\right]+p_{5}\left[c_{2}\right]+p_{6}[\mathbf{0}]$ would also work, because we may distribute one object net's tokens arbitrarily between all object nets of this same type. Also note that $(\lambda, \rho)$ is not the only mode that enables the above event. If we take the other (empty) object net on place $p_{1}$, we would still be able to fire. The transition $t_{1}$ in the object net is still enabled due to the single token from the object net on $p_{2}$.

Again interpreting the object nets as mobile agents and the system net as the environment, the firing of transition $t$ synchronously with transition $t_{1}$ of object net $N_{1}$ and $t_{2}$ of object net $N_{2}$, may be viewed as an interaction of the two agents represented by the different object nets. A object-autonomous event may then be viewed as an independent action of an agent, while a system-autonomous event may either be viewed as something that happens in the environment or as a movement of an agent. A synchronous event may be an interaction between several agents as above, or it might be an interaction of one or several agents with the environment. $\diamond$

Example 2.5. We now consider a mobile agent which moves from its home to the office where it can execute two different tasks in parallel before he leaves for the city. The example system shown in figure 3 can be expressed in rewriting logic [19]. The following specification is made in MAUDE syntax. All operators for the system level are prefixed by "sn" and "on" for the object net.

$\bmod$ ONS is 


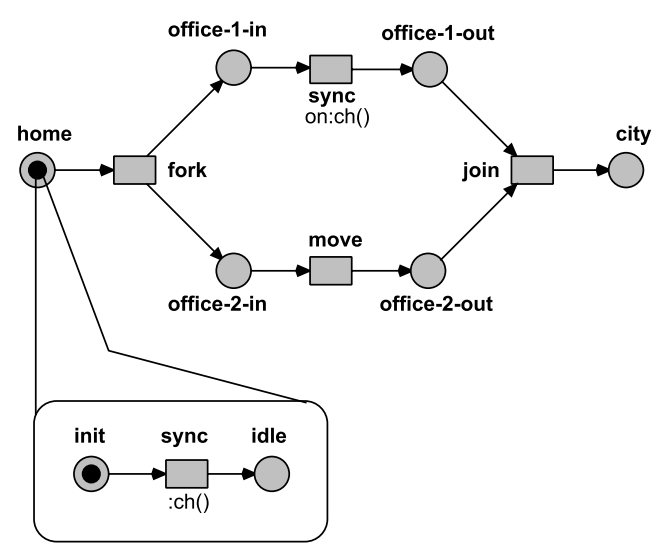

Figure 3: A simple object net system

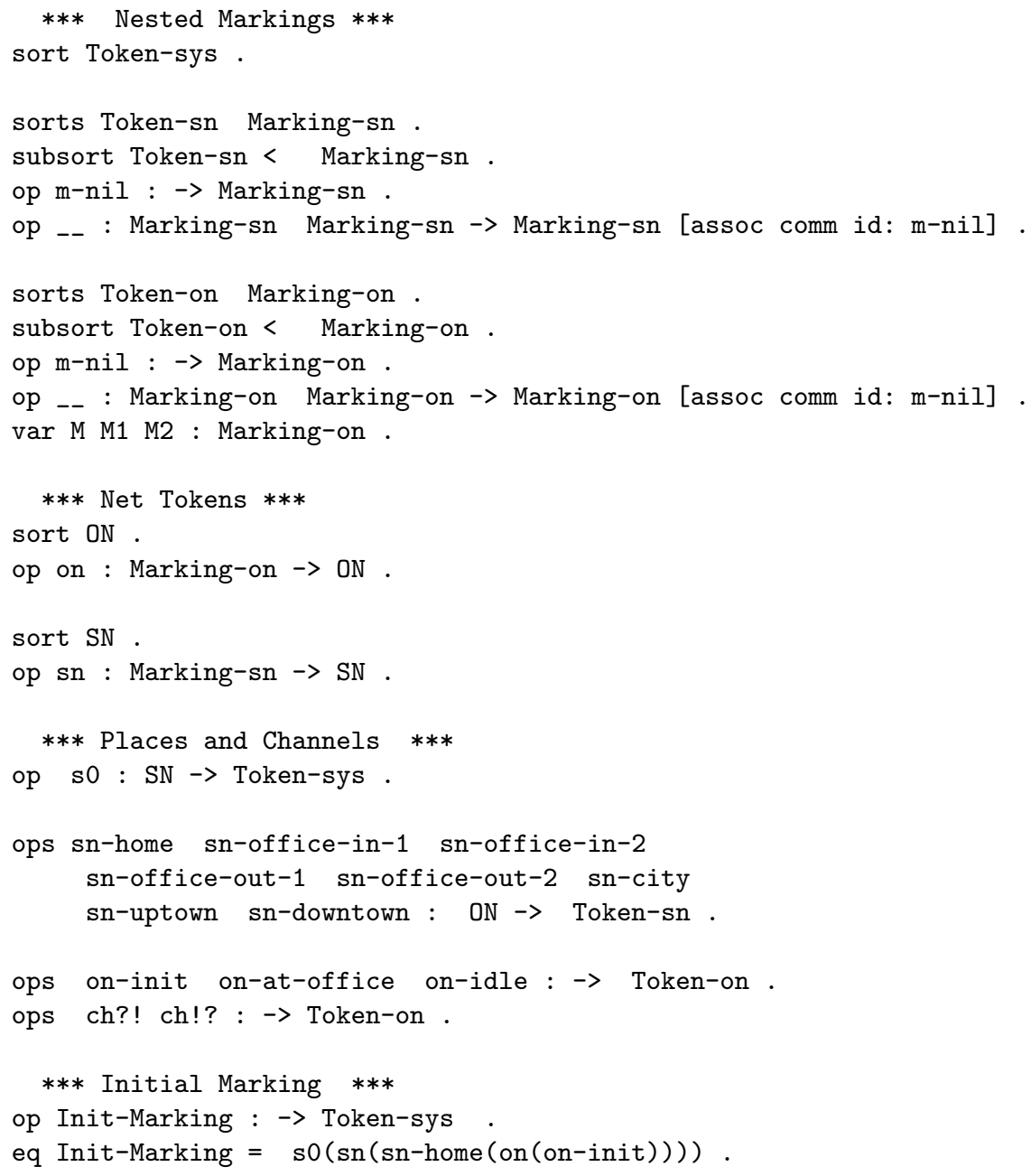




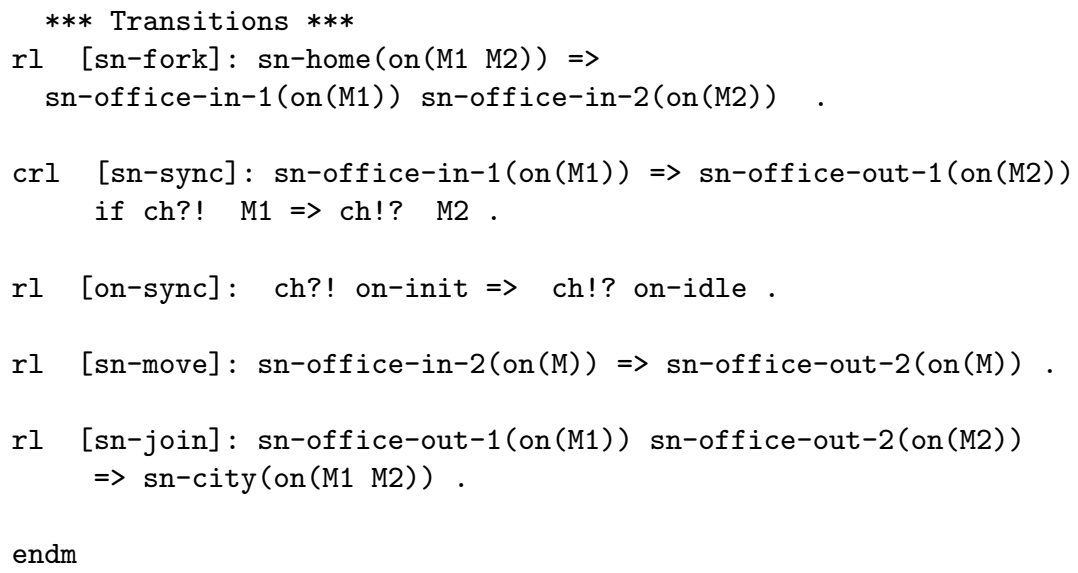

This specification allows a rewriting sequence (i.e. firing sequence) resulting in the successor marking so(sn(sn-city(on(on-idle)))):

Maude> rew Init-Marking .

rewrites: 6 in $0 \mathrm{~ms} \mathrm{cpu}$ (5ms real) ( rewrites/second)

result Token-sys: $\mathrm{s} 0(\mathrm{sn}(\mathrm{sn}$-city $($ on $($ on-idle $)))$ ) Maude>

MAUDE provides a generic LTL model checker [2]. The user only has to provide the basic properties. For our example we define two properties: officezout and terminated. The first proposition is true whenever the place sn-office-out-2 is marked and the second when the place sn-city is marked.

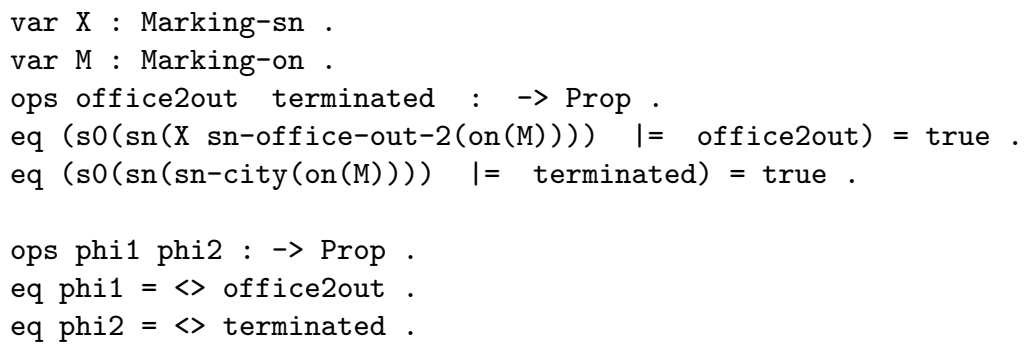

In this specification we have the two LTL formulae $\phi_{1}$ and $\phi_{2}$. The formula phi1 = <> office2out is the representation of $\phi_{1}=\diamond$ office2out, which expresses that the mobile agent will sometimes be at the place sn-office-out-2. The satisfiability of the logical atoms is given as equations:

$$
\text { eq }(s o(\operatorname{sn}(X \text { sn-office-out-2(on(M)))) } I=\text { office2out })=\text { true }
$$

Model-checking the state spaces reveals the correctness of $\phi_{1}$ :

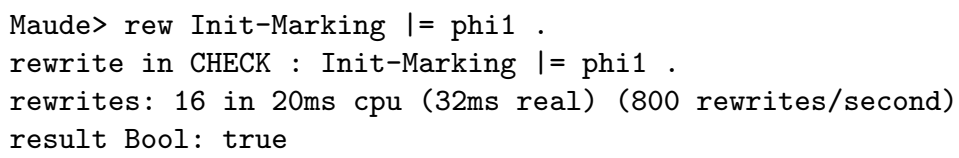


The formula phi2 $=<>$ terminated (for $\phi_{2}=\diamond$ terminated) describes, together with the equation

$$
\text { eq }(\operatorname{so}(\operatorname{sn}(\operatorname{sn}-\operatorname{city}(o n(M)))) I=\text { terminated })=\text { true },
$$

that the place sn-city will eventually be occupied by the mobile agent. It is maybe not obvious at first sight that there is one trace which refutes the property: When firing the transition fork the token on on-init might not be assigned to the object net on place sn-office-1-in and therefore the synchronisation is disable. The model checking processes reveals this counter example:

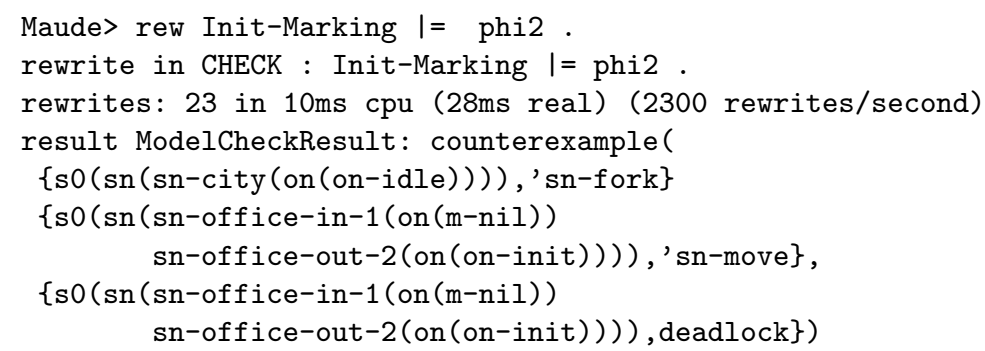

This example illustrates the potential of LTL model checking in the context of mobile agents modelled with object nets.

\section{Boundedness and Safe Eos}

Boundedness is the problem to decide whether there are only finitely many reachable markings. A p/t net is called $n$-safe with $n \in \mathbb{N}$ if in every reachable marking there are at most $n$ tokens on each place: $\forall m \in R S\left(\mathbf{m}_{0}\right): \forall p \in P: m(p) \leq n$. A net that is $n$-safe for some $n$ is also called bounded. The following property is well-known for $\mathrm{p} / \mathrm{t}$ nets.

Lemma 3.1. The set of reachable markings of a $p / t$ net $N$ is finite iff $N$ is $n$-safe for some $n$.

Proof. If $N$ is $n$-safe then $\left|R S\left(\mathbf{m}_{0}\right)\right| \leq(n+1)^{|P|}$. If the set of reachable markings is finite then $N$ is $n$-safe for $n:=\max \left\{\mathbf{m}(p) \mid p \in P, \mathbf{m} \in R S\left(\mathbf{m}_{0}\right)\right\}$.

A p/t net is called safe if it is 1-safe. Therefore, each reachable marking of a safe net is a set and we have $\left|R S\left(\mathbf{m}_{0}\right)\right| \leq 2^{|P|}$, i.e. the number of subsets of $P$.

In the following we consider bounded object nets. Reachability is decidable whenever the object nets are bounded. An Eos is $N$-bounded if $\Pi_{N}^{2}(\mu)$ is bounded in each reachable marking $\mu$. An Eos is semi-bounded if it is bounded for all object nets $N \in \mathcal{N}$. The reachability problem is decidable for a semi-bounded Eos ([8, Thm. 3.4).

Note that it is possible that an Eos is $N$-bounded but $N$ (considered as a p/t net in isolation) is not bounded for the initial marking $\Pi_{N}^{2}\left(\mu_{0}\right)$. Any unbounded object net where each transition is synchronised with a dead system net transition is an example.

In [14] we identified four different variants of safeness - named safe(1), safe(2), safe(3), and safe $(4)$ - where $\operatorname{safe}(i+1)$ implies safe $(i)$, but not vice versa. On $\mathrm{p} / \mathrm{t}$-like Eos all these variants coincide.

Definition 3.2. Let $O S$ be an Eos.

- OS is safe(1) iff all reachable markings are sets.

$$
\forall \mu \in R S(O S): \forall \widehat{p}[M]: \mu(\widehat{p}[M]) \leq 1
$$


- OS is safe(2) iff for all reachable markings there is most one token on each system net place:

$$
\forall \mu \in R S(O S): \quad \forall \widehat{p} \in \widehat{P}: \Pi^{1}(\mu)(\widehat{p}) \leq 1
$$

- OS is safe(3) iff for all reachable markings there is most one token on each system net place and each net-token is safe:

$$
\begin{aligned}
\forall \mu \in R S(O S): & \forall \widehat{p} \in \widehat{P}: \Pi^{1}(\mu)(\widehat{p}) \leq 1 \wedge \\
& \forall N \in \mathcal{N}: \forall p \in P_{N}: \forall \widehat{p}[M] \leq \mu: M(p) \leq 1
\end{aligned}
$$

- OS is safe(4) iff for all reachable markings there is most one token on each place (w.r.t. projections):

$$
\begin{aligned}
\forall \mu \in R S(O S): & \forall \widehat{p} \in \widehat{P}: \Pi^{1}(\mu)(\widehat{p}) \leq 1 \wedge \\
& \forall N \in \mathcal{N}: \forall p \in P_{N}: \Pi_{N}^{2}(\mu)(p) \leq 1
\end{aligned}
$$

We simply say that $O S$ is a safe EOS if any of the above properties hold.

In the following we concentrate on the variant of safe(3) Eos, since it turned out that reachability for safe(1) or safe(2) Eos is undecidable and an algorithm for the reachability problem for safe(3) Eos will also work with safe(4) Eos. For generalised state machines safe(3) is even equivalent to safe(4) (cf. [14]).

In contrast to $\mathrm{p} / \mathrm{t}$ nets not all notions of safeness are equivalent to finiteness of the state space: The state space of safe(1) or safe(2) Eos is infinite in the general case. Finiteness only holds for safe(3) and safe(4) Eos:

Theorem 3.3 (14). If an Eos is safe(3) or safe(4) then its set of reachable markings is finite.

By Theorem 3.3 we know that (strongly) safe Eos have finite state spaces, but compared to the state spaces of safe $\mathrm{p} / \mathrm{t}$ nets they may become quite large: For 1 -safe $\mathrm{p} / \mathrm{t}$ nets it is known that whenever there are $n$ places, then the number of reachable states is bound by $O\left(2^{n}\right)$. In the proof of Thm. 3.3 however, we have seen that whenever the number of places in the system net and in all object nets is bound by $n$, then the number of reachable states is in $O\left(2^{n^{2}}\right)-$ a quite drastic increase. This combinatorical explosion makes it in general very hard to represent the state space explicitly. In the following we characterise this kind of blow-up in complexity theoretical terms.

\section{Complexity of LTL Model Checking}

It is a known fact that most interesting questions about the behaviour of classical 1-safe $\mathrm{p} / \mathrm{t}$ nets like liveness, deadlock-freedom, and reachability are PSPACE-hard (see [3]). This follows from the fact, first observed in [7, that a 1-safe $\mathrm{p} / \mathrm{t}$ net of size $\mathcal{O}\left(n^{2}\right)$ can simulate a linear bounded automaton starting on an empty tape of size $n$. Since the net can furthermore be constructed in polynomial time, hardness results concerning linear bounded automata carry over to 1 -safe $\mathrm{p} / \mathrm{t}$ nets. From there they directly carry over to safe Eos, since 1 -safe $\mathrm{p} / \mathrm{t}$ nets can be seen as a special kind of safe(4) Eos, which are also safe(3), safe(2), and safe(1). Thus it is for instance PSPACE-hard to decide reachability and liveness for safe Eos.

The more interesting question is therefore, if polynomial space suffices and, if so, devise algorithms for these problems.

In [14] we showed that many problems on safe(3) and safe(4) Eos can be decided in polynomial space. More explicitly we showed that Theorem 4.1 below holds. Among other problems 
reachability can be expressed as an LTL formula and is thus PSPACE-complete. Liveness can not be expressed as an LTL formula and we will address the problem to decide liveness in the outlook. Our construction in [14 is an adjustment of the construction given in [3] for 1-safe $\mathrm{p} / \mathrm{t}$ nets. In 14 we explicitly pointed out the similarities. Here we take a more algorithmic oriented point of view, but we want to emphasize that the following result heavily relies on ideas from [3] and also 25.

In the following we will uses LTL formulae to express a property like reachability with a formula $\phi$. A net is then said to satisfy $\phi$ if all its runs satisfy $\phi$. A run of a net is just a sequence of markings. To be able to express properties of a net with LTL formulas the set of atomic propositions should be closely related to the markings. To achive this let $\widehat{p_{1}}, \ldots, \widehat{p_{n}}$ be the system net's places, let $N_{1}, \ldots, N_{n}$ be the object nets with $d\left(\widehat{p}_{i}\right)=N_{i}$ for all $i$, and let $p_{N_{i}, 1}, \ldots, p_{N_{i}, m_{i}}$ be the places of the object net $N_{i} \cdot{ }^{5}$ We now define

$$
\text { prop }:=\left\{\begin{array}{l}
\widehat{p_{1}}[], \widehat{p_{1}}\left[p_{N_{1}, 1}\right], \ldots, \widehat{p_{1}}\left[p_{N_{1}, m_{1}}\right], \\
\ldots, \\
\widehat{p_{n}}[], \widehat{p_{n}}\left[p_{N_{n}, 1}\right], \ldots, \widehat{p_{n}}\left[p_{N_{n}, m_{n}}\right]
\end{array}\right\}
$$

as the set of atomic propositions for the LTL formulae. A marking is a subset of prop and a computation (resp. a run) is a sequence of subsets of prop. Here $\widehat{p_{1}}\left[p_{N_{1}, 1}\right]+\widehat{p_{2}}[]$ describes that the object net $N_{1}$ resides on the system net place $\widehat{p_{1}}$ and that an empty object net of type $N_{2}$ resides on the place $\widehat{p_{2}}$. The place $p_{N_{1}, 1}$ of the object net $N_{1}$ is marked. ${ }^{6}$

In the following we want to show the following theorem:

Theorem 4.1. Given a safe(3) or safe(4) Eos $N$ and an LTL formula $\phi$ checking whether $N$ satisfies $\phi$ can be done in polynomial space in the size of $N$ and $\phi$, that is, there is a polynomial $p$ independent of $N$ and $\phi$ such that the algorithm uses $O(p(|N|+|\phi|))$ space.

Since reachability can be expressed as an LTL formula we have the following corollary:

Corrolary 4.2. The reachability problem for safe(3) and safe(4) EOS is PSPACE-complete.

We need the following important result:

Theorem 4.3. Given an LTL formula $\phi$, one can build a finite automaton $A_{\phi}$ and a Büchi automaton $B_{\phi}$ such that $L\left(A_{\phi}\right) \cup L_{\omega}\left(B_{\phi}\right)$ is exactly the set of computations satisfying the formula $\phi$.

The proof of Theorem 4.3 exceeds the scope of this paper (see [3] and 25] for details). Here it suffices to know the following two facts: First, the states of $A_{\phi}$ are sets of subformulae of $\phi$ and the states of $B_{\phi}$ are pairs of sets of subformulae of $\phi$, thus they both may have exponentially many states in $|\phi|$ and must not be completly constructed if we want to prove Theorem 4.1 above. Yet note that a single state has polynomial size in $|\phi|$. Second, given two states $q_{1}$ and $q_{2}$ of $A_{\phi}$ or $B_{\phi}$ and a marking $\mu$ (which is a symbol of the alphabet of $A_{\phi}$ resp. $B_{\phi}$ ), it is possible to decide in polynomial space (in $|\phi|)$ if a transition from $q_{1}$ to $q_{2}$ with label $\mu$ exists. In what follows we will not need the automata $A_{\phi}$ and $B_{\phi}$, but the automata $A_{\neg \phi}$ and

\footnotetext{
${ }^{5}$ Note that in general some places of the system net might be typed with the same object net. In that case $N_{i}=N_{j}$ for some $i$ and $j$ and also $p_{N_{i}, k}=p_{N_{j}, k}$ for all $1 \leq k \leq m_{i}=m_{j}$.

${ }^{6}$ Note that with this interpretation some subsets of prop are useless. For example the set $\left\{\widehat{p_{1}}[], \widehat{p_{1}}\left[p_{N_{1}, 1}\right]\right\}$ describes the same marking as $\left\{\widehat{p_{1}}\left[p_{N_{1}, 1}\right]\right\}$, namely that the net $N_{1}$ resides on the place $\widehat{p_{1}}$ and that its first place is marked. This is only relevant for the given formula $\phi$. In general, in a conjunction of the form $\widehat{p_{i}}[] \wedge \widehat{p_{i}}\left[p_{N_{i}, k}\right] \wedge \ldots$ the conjunct $\widehat{p_{i}}[]$ can be deleted without loss. This is possible in time $O(|\phi|)$. If the formula is correctly given in the first place, no further problems arise.
} 


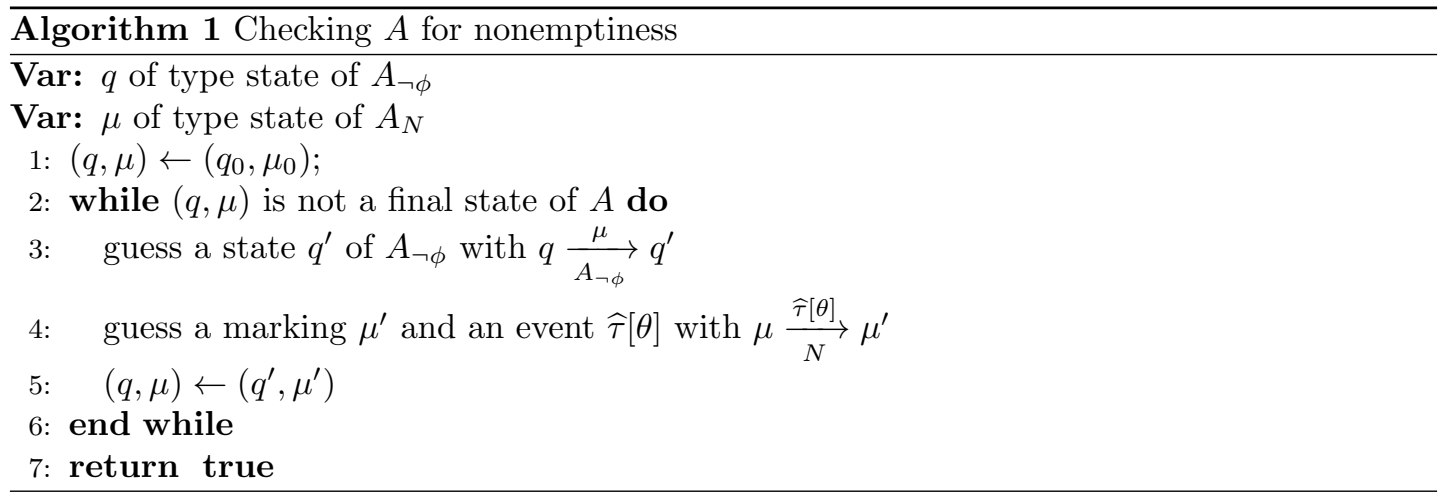

$B_{\neg \phi}$ instead. Below it will become clear why, but at first we will need two further automata, $A_{N}$ and $B_{N}$, obtained from the safe Eos $N$, whose set of states correspond to the reachable markings of the net $N$. The initial state of $A_{N}$ and $B_{N}$ is the initial marking and the transition relation $\delta_{N}$ (again the same for $A_{N}$ and $B_{N}$ ) contains the triple $\left(\mu_{1}, \mu_{1}, \mu_{2}\right)$ of markings if $\mu_{2}$ can be reached from $\mu_{1}$ via some event $\widehat{\tau}[\theta] \in \Theta$. Thus $A_{N}$ and $B_{N}$ correspond closely to the reachability graph and indeed only differ from it in the edges' labels. The set of final states of $A_{N}$ is the set of deadlocked reachable markings of $N$ and the set of final states of $B_{N}$ contains all reachable markings of $N$, thus $L\left(A_{N}\right)$ is the set of all finite and $L_{\omega}\left(B_{N}\right)$ is the set of all infinite runs of $N$. Note that $A_{N}$ and $B_{N}$ may have exponentially many states in $|N|$ and we again can not construct them completly.

We now define two automata $A$ and $B$ with the usual construction to be the product automata of $A_{\neg \phi}$ and $A_{N}$, resp. of $B_{\neg \phi}$ and $B_{N}$. Then $L(A)=L\left(A_{\neg \phi}\right) \cap L\left(A_{N}\right)$ and $L_{\omega}(B)=$ $L_{\omega}\left(B_{\neg \phi}\right) \cap L_{\omega}\left(B_{N}\right)$ holds $^{7}$ and $L(A) \cup L_{\omega}(B)$ is the set of runs of $N$ that do not satisfy $\phi$. The question whether $N$ satisfies $\phi$, i.e. if all of $N$ 's runs satisfy $\phi$, is thus reduced to the question if $L(A)$ and $L_{\omega}(B)$ are both empty.

To prove Theorem 4.1 we now devise a nondeterministic algorithm for the nonemptiness problem of $A$ and $B$. By Savitch's Theorem [21] a deterministic algorithm follows, and by reversing its answer we obtain the sought algorithm.

Apart from using nondeterminism we also make use of another trick: Since $A$ and $B$ may have exponentially many states in $|N|$ and $|\phi|$, we construct them on the fly. We start with the initial state as the current state $(q, \mu)$, repeatedly guess a next state $\left(q^{\prime}, \mu^{\prime}\right)$, check that $\left(q^{\prime}, \mu^{\prime}\right)$ is indeed reachable from $(q, \mu)$ via some transition and, if so, update the current state. In the case of $A$, we know that $L(A)$ is nonempty, if we reach a final state. We thus return true in this case. Algorihm 1 is outlined below and is only a minor modification from the algorithm given in 3 .

The case of $B$ is only slightly more complicated. Since $L_{\omega}(B)$ is nonemtpy if and only if a final state is visited infinitly often, i.e. if there is a reachable final state $q$ such that there is a loop from $q$ to itself, we proceed as in Algorithm 1 but if we reach a final state, we guess that this final state will be revisited or not. This guess is then checked in a similar way as Algorithm 1 checks for any final state. See [3] for details.

Why are these algorithms polynomial space algorithms (in $|N|$ and $|\phi|)$ ? At first we note that only a constant number of states need to be saved and that the states of $A_{\neg \phi}$ (resp. $B_{\neg \phi}$ )

\footnotetext{
${ }^{7}$ In general it is not the case that the product of two Büchi automata accepts the intersection of the languages, but it holds here.
} 
and $A_{N}\left(B_{N}\right)$ have polynomial size in $|\phi|$ and $|N| .^{8}$ Since $A$ and $B$ have been constructed with the product automata construction we actually need to check if a transition is possible in $A_{\neg \phi}$ and $A_{N}$ to check if one is possible in $A$ (and similar for $B$ ). For $A_{\neg \phi}$ (and $B_{\neg \phi}$ ) this follows from the construction of $A_{\neg \phi}$ which we have not shown here. (See for example [25].) In the algorithm one only needs to store a state of $A_{\neg \phi}$ and a symbol of the alphabet (which is a marking of $N$ ) of $A_{\neg \phi}$. Then one can check similar to Algorithm 1 if a final state can be reached. This can be done in polynomial space. See [25] for more details.

To check if a transition in $A_{N}$ or $B_{N}$ is possible, we need to check if, given two markings $\mu, \mu^{\prime} \in \mathcal{M}$ and an event $\widehat{\tau}[\theta] \in \Theta$, the relation $\mu \underset{N}{\stackrel{\widehat{\tau}[\theta]}{\longrightarrow}} \mu^{\prime}$ holds.

For this we first check that $\widehat{\tau}[\theta]$ is indeed an event. Using the synchronisation labeling this can easily be done in space polynomial in $|N|$, indeed we only need constant space for this. Now we nondeterministicallys guess ${ }^{9}$ a mode $(\lambda, \rho) \in \mathcal{M}^{2}$, which is in $O\left(|N|^{2}\right)$ and check deterministically if the event $\widehat{\tau}[\theta]$ is enabled in $\mu$ for this mode, that is we check that $\lambda \leq \mu$ and $\phi(\widehat{\tau}[\theta], \lambda, \rho)$ holds. This is both possible in polynomial space, where the former is obvious and for the later we only need to compare partial markings of $\lambda$ and $\rho$ with the presets and postsets of certain nets. At last we check that $\mu^{\prime}=\mu-\lambda+\rho$ holds, which again is possible in polynomial space. We thus have an algorithm to nondeterministically decide $\mu \stackrel{\widehat{\tau}[\theta]}{N} \mu^{\prime}$ in polynomial space. By Savitch's Theorem [21] a deterministic algorithm follows.

Finally we need to be able to check if a state in $A$ (resp. $B$ ) is a final state, that is we need to check for final states in $A_{\neg \phi}, B_{\neg \phi}, A_{N}$ and $B_{N}$. For $A_{\neg \phi}$ and $B_{\neg \phi}$ one by their definition only needs to do some syntactic checks on the states, which are actually sets of subformulas of $\phi$. This is easily possible in polynomial space. To check for final states in $B_{N}$ is also simple, since the set of final states of $B_{N}$ equals its set of states. At last to check if a state of $A_{N}$ is a final state, we need to check if a marking $\mu$ of $N$, which is a state of $A_{N}$, is a deadlock, that is, we have to check that no event is enabled in $\mu$. Above we have devised an algorithm which given $\mu$ guesses $\mu^{\prime}$ and an event and then checks if $\mu^{\prime}$ is reachable from $\mu$ with this event, that is the algorithm checks that $\mu$ is not a deadlock. Since this algorithm is deterministic we can just reverse the answer and have an algorithm to check if $\mu$ is a deadlock.

Putting it all together we have proven Theorem 4.1.

\section{Conclusion and Outlook}

In this paper we discussed the concept of safeness for Elementary Object Nets (EOS). We have shown that reachability is PSPACE-complete for safe(3) and safe(4) EOS and that in fact every property that can be expressed in LTL is decidable in PSPACE for safe(3) and safe(4) EOS. We also stressed the usefullness of EOS as a modelling tool for mobile objects or agents.

Not all interesting properties can be expressed in LTL, though, and liveness is a particularly interesting example. Liveness can be expressed in CTL. However, CTL formulas need to be treated differently than the LTL formulas discussed above. In 3 it is shown, that the modelchecking problem for CTL and 1-safe p/t nets is in PSPACE, too. It seems to us that these results, with slight modifications, carry over to safe(3) and safe(4) Eos, too. This and the related case for safe(1) and safe(2) Eos will be further investigated in a forthcoming paper.

Appart from this a closer look at the LTL case might be interesting. The model checking problem for many problems might indeed be far easier to solve than the general case presented

\footnotetext{
${ }^{8}$ To be more concrete: The states of $A_{\phi}$ and $B_{\phi}$ have size quadratic in $|\phi|$ and the states of $A_{N}$ and $B_{N}$ are quadratic in $|N|$.

${ }^{9}$ Note that we have already guessed $\mu^{\prime}$ and $\widehat{\tau}[\theta]$, which also only have polynomial size in the size of the net.
} 
here. Also the case for safe(4) Eos - even if PSPACE-complete, too - might be algorithmically easier to solve than the case for safe(3) Eos from a practitioners point of view. This might be especially interesting with applications and therefore the need of an efficient implementation of the LTL model checking algorithm above in mind.

\section{References}

[1] Marek A. Bednarczyk, Luca Bernardinello, Wieslaw Pawlowski, and Lucia Pomello. Modelling mobility with Petri hypernets. In Josè Luiz Fiadeiro, Peter D. Mosses, and Fernando Orejas, editors, Recent Trends in Algebraic Development Techniques (WADT 2004), volume 3423 of Lecture Notes in Computer Science, pages 28-44. Springer-Verlag, 2004.

[2] Steven Eker, José Meseguer, and Ambarish Sridharanarayanan. The Maude LTL model checker. In Electronic Notes in Theoretical Computer Science, volume 71. Elsevier, 2002.

[3] Javier Esparza. Decidability and complexity of petri net problems - an introduction. In Wolfgang Reisig and Grzegorz Rozenberg, editors, Lectures on Petri Nets I: Basic Models, Advances in Petri Nets, volume 1491 of Lecture Notes in Computer Science, pages 374-428. Springer-Verlag, 1998.

[4] Serge Haddad and Denis Poitrenaud. Theoretical aspects of recursive Petri nets. In S. Donatelli and J. Kleijn, editors, Application and Theory of Petri Nets, volume 1639 of Lecture Notes in Computer Science, pages 228-247. Springer-Verlag, 1999.

[5] Kunihiko Hiraishi. $\mathrm{PN}^{2}$ : An elementary model for design and analysis of multi-agent systems. In Farhad Arbab and Carolyn L. Talcott, editors, Coordination Models and Languages, COORDINATION 2002, volume 2315 of Lecture Notes in Computer Science, pages 220-235. Springer-Verlag, 2002.

[6] Kathrin Hoffmann, Hartmut Ehrig, and Till Mossakowski. High-level nets with nets and rules as tokens. In Application and Theory of Petri Nets and Other Models of Concurrency, volume 3536 of Lecture Notes in Computer Science, pages 268 - 288. Springer-Verlag, 2005.

[7] N. D. Jones, L. H. Landweber, and Y. E. Lien. Complexity of some problems in petri nets. Theoretical Computer Science, 4:277-299, 1977.

[8] Michael Köhler. Reachable markings of object Petri nets. Fundamenta Informaticae, 79(3-4):401 $-413,2007$.

[9] Michael Köhler, Daniel Moldt, and Heiko Rölke. Modeling the behaviour of Petri net agents. In J. M. Colom and M. Koutny, editors, Application and Theory of Petri Nets, volume 2075 of Lecture Notes in Computer Science, pages 224-241. Springer-Verlag, 2001.

[10] Michael Köhler, Daniel Moldt, and Heiko Rölke. Modelling mobility and mobile agents using nets within nets. In W. v. d. Aalst and E. Best, editors, Application and Theory of Petri Nets, volume 2679 of Lecture Notes in Computer Science, pages 121-140. Springer-Verlag, 2003.

[11] Michael Köhler and Heiko Rölke. Concurrency for mobile object-net systems. Fundamenta Informaticae, 54(2-3), 2003.

[12] Michael Köhler and Heiko Rölke. Properties of Object Petri Nets. In J. Cortadella and W. Reisig, editors, Application and Theory of Petri Nets, volume 3099 of Lecture Notes in Computer Science, pages 278-297. Springer-Verlag, 2004.

[13] Michael Köhler-Bußmeier. Hornets: Nets within nets combined with net algebra. In Karsten Wolf and Giuliana Franceschinis, editors, Application and Theory of Petri Nets, volume 5606 of Lecture Notes in Computer Science, pages 243-262. Springer-Verlag, 2009.

[14] Michael Köhler-Bußmeier and Frank Heitmann. Safeness for object nets. Fundamenta Informaticae, 2010. To appear.

[15] Olaf Kummer, Roxana Dietze, and Manfred Kudlek. Decidability problems of a basic class of object nets. Fundamenta Informaticae, 79(3-4):295-302, 2008. 
[16] Charles Lakos. A Petri net view of mobility. In Formal Techniques for Networked and Distributed Systems (FORTE 2005), volume 3731 of Lecture Notes in Computer Science, pages 174-188. Springer-Verlag, 2005.

[17] Irina A. Lomazova. Nested Petri nets - a formalism for specification of multi-agent distributed systems. Fundamenta Informaticae, 43(1-4):195-214, 2000.

[18] Irina A. Lomazova, Kees M. van Hee, Olivia Oanea, Alexander Serebrenik, Natalia Sidorova, and Marc Voorhoeve. Nested nets for adaptive systems. In Application and Theory of Petri Nets and Other Models of Concurrency, Lecture Notes in Computer Science, pages 241-260. Springer-Verlag, 2006.

[19] José Meseguer. Conditional rewriting logic as a unified model of concurrency. Theoretical Computer Science, 96:73-155, 1992.

[20] Wolfgang Reisig and Grzegorz Rozenberg, editors. Lectures on Petri Nets I: Basic Models, volume 1491 of Lecture Notes in Computer Science. Springer-Verlag, 1998.

[21] W.J. Savitch. Relationship between nondeterministic and deterministic tape complexities. J. on Computer and System Sciences, 4:177-192, 1970.

[22] Rüdiger Valk. Modelling concurrency by task/flow EN systems. In 3rd Workshop on Concurrency and Compositionality, number 191 in GMD-Studien, St. Augustin, Bonn, 1991. Gesellschaft für Mathematik und Datenverarbeitung.

[23] Rüdiger Valk. Petri nets as token objects: An introduction to elementary object nets. In Jörg Desel and Manuel Silva, editors, Application and Theory of Petri Nets, volume 1420 of Lecture Notes in Computer Science, pages 1-25. Springer-Verlag, 1998.

[24] Rüdiger Valk. Object Petri nets: Using the nets-within-nets paradigm. In Jörg Desel, Wolfgang Reisig, and Grzegorz Rozenberg, editors, Advanced Course on Petri Nets 2003, volume 3098 of Lecture Notes in Computer Science, pages 819-848. Springer-Verlag, 2003.

[25] Moshe Vardi. An automata-theoretic approach to linear temporal logic. In F. Moller and G. Birtwistle, editors, Logics for Concurrency: Structure versus Automata, volume 1043 of Lecture Notes in Computer Science, pages 238-266. Springer-Verlag, 1996. 